DOI: 10.1515/awutp -2016-0003

\title{
QUANTIFICATION OF THE SOLAR RADIATIVE REGIME VARIABILITY BASED ON THE CLEARNESS INDEX
}

\author{
Sergiu Lucaciu, Robert Blaga, Nicoleta Stefu, Marius Paulescu \\ Physics Department, West University of Timisoara, V. Parvan Ave.4, 300223 Timisoara, Romania
}

\begin{tabular}{|l|l|}
\hline \multicolumn{1}{|c|}{ Article Info } & \multicolumn{1}{c|}{ Abstract } \\
\hline $\begin{array}{l}\text { Received: } 25.09 .2016 \\
\text { Accepted: } 30.11 .2016\end{array}$ & $\begin{array}{l}\text { The fluctuation of solar radiation at ground level represents a challenge in } \\
\text { modeling the time series of solar irradiance. A procedure for the quantification of } \\
\text { the instability of the solar radiative regime is reported. This procedure is based on } \\
\text { the clearness index, as the ratio of the horizontal solar irradiance measured at } \\
\text { Keywords: Clearness } \\
\text { index; solar radiation } \\
\text { variability. }\end{array}$ \\
$\begin{array}{l}\text { classifying the days from the radiative stability point of view are being introduced. } \\
\text { A procedure for classifying days according to their stability regime as stable, } \\
\text { variable and unstable is presented. }\end{array}$ \\
\hline
\end{tabular}

\section{Introduction}

In a given point, the solar radiation at ground level exhibits a continuous variation in time. This variation has a deterministic component generated by the rotation and revolution movements of the Earth and a fluctuating component due to weather pattern irregularities. Changes in solar irradiance at a point due to the passing clouds may be huge, exceeding $60 \%$ of the peak in seconds [1]. Thus, understanding the fluctuation of solar radiation at ground represents a key issue in modeling the time series of solar irradiance at the ground level.

Different facets of solar radiation variability were studied from different perspectives. For example, Tomson and Tamm report in [2] a study on solar irradiance variability based on oneminute data series. A stability criterion for the classification of days as clear or cloudy and alternating has been proposed. The dynamic behaviour of diffuse radiation was reported by Tomson in [3], showing that, in conditions of alternating clouds, global radiation and its diffuse 
fraction do not change synchronously. Diffuse fraction is more stable than the global radiation at alternating clouds.

The above study investigated the solar irradiance data series, including its deterministic component. The traditional approach for isolating the stochastic component of solar irradiance is by means of the instantaneous clearness index [4]:

$$
k_{t}=G / G_{\text {ext }}
$$

where $G$ and $G_{\text {ext }}$ are the horizontal solar irradiance measured at ground level and estimated at extraterrestrial level, respectively. Thus clearness index accounts for all meteorological influence, being a measure of the atmosphere transparency. A review of the statistical behavior of the solar radiation components based on clearness index can be read in [5].

The clearness index is a usual a quantity for assessing the variability in solar irradiance data series. For example, Woyte et al. [6] introduced the method of localized spectral analysis for the evaluation of cloud-induced fluctuations in solar irradiance. The authors demonstrate how the localized spectral analysis allows the identification and classification of fluctuations in time series of the instantaneous clearness index according to their persistence.

This paper is focused on developing a procedure for the quantification of the solar radiative regime based on the clearness index. The proposed procedure is inspired by Tomson et al. in [7]. Two improvements are brought over the study reported in [7]: (1) the solar radiative regime is studied in terms of clearness index instead of global solar irradiance. The clearness index removes the determinist component of the solar irradiance, keeping only the fluctuating component; (2) New measures for quantifying the variability of the solar radiative regime are defined.

\section{Database}

High-frequency (30 seconds resolution or better) solar data are required for studying the cloud-induced variability in solar radiation [8]. Woyte et al. [6] used up to 1 second irradiance measurements in Germany and Belgium; Perez et al. [9] used the 20 seconds measured irradiance data from the ARM network in northern Oklahoma and southern Kansas. In this study, global and diffuse solar irradiance ( $G$ and $G_{d}$, respectively) recorded in 2010 on the Solar 
Platform of the West University of Timisoara [10] are post-processed in order to compute the clearness index. On the Solar Platform measurements are performed all day long at equal time intervals of 15 seconds. DeltaOHM LP PYRA 02 first class pyranometers which fully comply with ISO 9060 standards and meet the requirements defined by the World Meteorological Organization are employed. The sensors are integrated into an acquisition data system based on National Instruments PXI Platform including a PXI-6259 data acquisition board optimized for high accuracy. The system operation is relying on a LabVIEW application.

The climate of Timisoara is temperate continental with an Ivanov index of continentality $130.9 \%[11]$.

\section{Results and discussions}

The solar radiative regime defines generically the variation of global solar irradiance during a given time period. The solar radiative regime is straightforwardly related to the state of the sky. Thus, the natural measure of the state of the sky, the total cloud amount, can be applied to the solar radiative regime too. Total cloud amount $C$ represents the fraction of the celestial vault covered by clouds. $C$ is essentially an instantaneous quantity. Nevertheless, $C$ average over a given period may be computed. On the daily level, the average of $C$ can be used for stratifying the days in respect to the state of the sky [12]. This is justified by the observed persistence of $C$.

In this paper we propose a measure of the solar radiative regime based on the clearness index, which consists on the following steps: The day length is divided in equal time intervals $\Delta \tau$ of 10 minutes. Each time interval $\Delta \tau$ contains a series of 40 values of the clearness index (obtained from the 15 seconds solar irradiance values measured on the Solar Platform). The difference between two different consecutive values of the clearness index defines a new time series, namely the time series of the clearness index increments:

$$
\Delta k_{t, j}=k_{t, j}-k_{t, j-1}
$$

Based on this series we define the stability index of a time interval $\Delta \tau$ :

$$
S_{i}=\sum_{j} \Delta k_{t, j}
$$




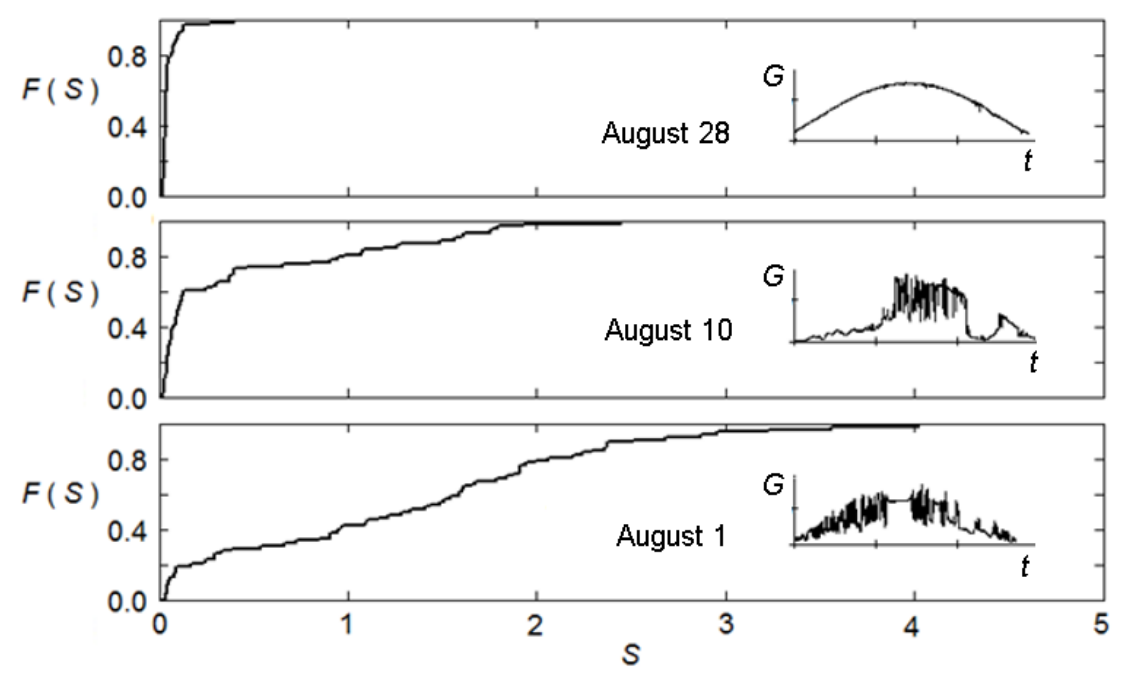

Figure 1. Cumulative distribution of the stability index in three different days from August 2010. The inner window presents the measured global solar irradiance.

Equation (3) introduces a new measure of the solar radiative regime within $\Delta \tau$. This is illustrated in Fig. 1, where the cumulative distribution of the stability index is plotted for three different days from August 2010: August 28, with a fully stable solar radiative regime (see the inner window), August 10, characterized by a moderate variability and August 1, with a fully unstable solar radiative regime. Figure 1 shows that the cumulative distribution of the stability index experiences different trajectories: a steep one in August 28, with a moderate slope in August 10 and with a slight slope in August 1. Therefore, in a given day, as the instability of the solar radiative regime increases, the slope of the cumulative distribution of the stability index decreases. These observations substantiate the fact that the cumulative distribution of the stability index represents a suitable measure for the variability of the radiative solar regime of a day. However, the cumulative distribution of the stability index gives a qualitative assessment of the solar radiative regime in a given day. In order to stratify the days over the variability of the solar radiative regime, quantitative criteria are required. For this, we propose the following procedure:

First, in order to categorize the different time intervals $\Delta \tau$ in respect with the stability of the solar radiative regime, we have established empirically the next criterion: if the stability index $S$ is greater than 0.89 , then the solar radiative regime in the time interval $\Delta \tau$ is unstable; otherwise the solar radiative regime $\Delta \tau$ is stable. The degree of instability of a given day was 
defined as the ratio between the number $N_{u}$ of unstable intervals $\Delta \tau$ and the total number of the intervals $N$ :

$$
P=\frac{N_{u}}{N} \times 100[\%]
$$

Based on the degree of instability $P$ a quantitative criterion for stratifying the days in respect to the solar radiative variability can be defined:

- $\quad P_{i}<5 \%$ - a day with a stable radiative solar regime

- $\quad 5 \%<P_{i}<50 \%$ - a day with a variable radiative solar regime

- $\quad P_{i}<50 \%$ - a day with unstable radiative solar regime

In this definition the thresholds are in accordance with [7].

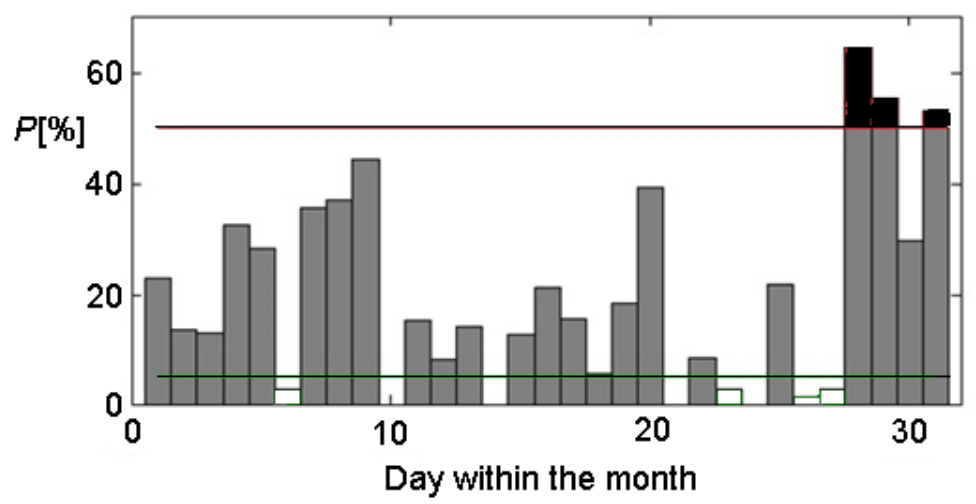

Figure 2. The degree of instability of the solar radiative regime in all the days of August 2010. The lower horizontal line (green) marks the stability threshold while the upper horizontal line marks the instability threshold.

\section{Conclusions}

The degree of instability of the solar radiative regime is an important issue when it comes to modeling the solar radiation at ground level. In order to take steps forward in classifying the days based on the stability of their radiative regime, new quantities were introduced: the stability index, as the sum of the clearness index increments in a time interval, and the degree of instability of a given day, as the ratio between the number of unstable intervals and the total number of the intervals in the day. By using these measures, the solar radiative regime of the days of a month, obtained from experimental data, was classified as stable, variable and unstable. 


\section{References}

[1] Mills A, Ahlstrom M, Brower M, Ellis A, George R, Hoff T, Kroposki B, Lenox C, Miller N, Milligan M, Stein J, Wan Y-h. Understanding Variability and Uncertainty of Photovoltaics for Integration with the Electric Power System. IEEE Power Energy M 2011; 9(3): 33 - 41.

[2] Tomson T, Tamm G (2006) Short-term variability of solar radiation. Solar Energy 80:600606

[3] Tomson T (2014) Dynamic behaviour of diffuse solar radiation. Theoretical and Applied Climatology 117:399-402

[4] Liu BY, Jordan RC (1960) The interrelationship and characteristic distribution of direct, diffuse and total solar radiation. Solar Energy 4:1-19.

[5] Tovar-Pescador J (2008) Modelling the Statistical Properties of Solar Radiation and Proposal of a Technique Based on Boltzmann Statistics. In V. Badescu (ed). Modeling Solar Radiation at the Earth's Surface, Springer, Berlin, 2008, p. 55

[6] Woyte A, Belmans R, Nijs J (2007) Fluctuations in instantaneous clearness index: Analysis and statistics. Solar Energy 81: 195-206.

[7] Tomson T, Russak V, Kallis A (2008) Dynamic Behavior of Solar Radiation. Ch. 10 in Nodeling Solar Radiation at the Earth Surface (Badescu V, Ed.), Springer, Berlin, pp. 257282.

[8] Lave M, Kleissl J, Arias-Castro E (2012) High-frequency irradiance fluctuations and geographic smoothing. Solar Energy 86 (8): 2190-2199.

[9] Perez R, Kivalov S, Schlemmer J, Hemker Jr. K, Hoff TE (2012) Short-term irradiance variability: preliminary estimation of station pair correlation as a function of distance. Solar Energy 86 (8): 2170-2176.

[10] SP (2016) Solar Radiation Monitoring Station of West University of Timisoara, Romania. http://solar.physics.uvt.ro/srms

[11] Badescu V (1999) Correlations to estimate monthly mean daily solar global irradiation: application to Romania, Energy, 24:883-893.

[12] Paulescu M, Badescu V (2011) New approach to measure the stability of the solar radiative regime. Theoretical and Applied Climatology 103: 459-70. 